\title{
Synthesis and Biological Evaluation of New Substituted 3-[4-(Phenylsulfonamido)benzoyl]-2H-1-benzopyran-2-one Derivatives as $\alpha$-Glucosidase Inhibitors
}

\author{
Yu-ling Wang, ${ }^{1}$ Ting-jian Zhang, ${ }^{1}$ Jing-wei Liang, ${ }^{1}$ Fan-hao Meng, ${ }^{1}$ and Shao-jie Wang ${ }^{2}$ \\ ${ }^{1}$ School of Pharmacy, China Medical University, Shenyang, Liaoning 110001, China \\ ${ }^{2}$ Key Laboratory of Structure-Based Drugs Design \& Discovery of Ministry of Education, \\ Shenyang Pharmaceutical University, Shenyang, Liaoning 110016, China \\ Correspondence should be addressed to Fan-hao Meng; fhmeng@cmu.edu.cn and Shao-jie Wang; sjwang_99@yahoo.com.cn
} Received 17 January 2014; Accepted 10 March 2014; Published 6 April 2014

Academic Editor: Gabriel Navarrete-Vazquez

Copyright (C) 2014 Yu-ling Wang et al. This is an open access article distributed under the Creative Commons Attribution License, which permits unrestricted use, distribution, and reproduction in any medium, provided the original work is properly cited.

A series of new substituted 3-[4-(phenylsulfonamido)benzoyl]-2H-1-benzopyran-2-one derivatives bearing groups methoxy, tertbutyl, and atoms of halogens at the para-position of the A-ring were synthesized and in vitro biological activities were evaluated as nonsugar $\alpha$-glucosidase inhibitors. Most of the test compounds demonstrated significant $\alpha$-glucosidase inhibitory activity relative to that of Acarbose $\left(\mathrm{IC}_{50}=29.26 \mu \mathrm{M}\right)$. The para-substitution with a methoxy group or halogens could notably increase the potency. Compounds 17, 18, and 23, with $\mathrm{IC}_{50}$ values of $0.025 \mu \mathrm{M}, 0.014 \mu \mathrm{M}$, and $0.018 \mu \mathrm{M}$, respectively, may be of significance for the further development of new nonsugar $\alpha$-glucosidase inhibitors.

\section{Introduction}

$\alpha$-Glucosidase (EC 3.2.1.20), located in the brush-border surface membrane of intestinal cells, is a key enzyme catalyzing the final step in the digestive process of carbohydrates into glucose [1]. This enzyme has drawn a special interest in the pharmaceutical research community because the inhibition of its catalytic activity can retard the liberation of glucose from dietary complex carbohydrates and delay glucose absorption, resulting in reduced postprandial plasma blood glucose level and suppression of postprandial hyperglycemia [2]. Thus, $\alpha$-glucosidase inhibitors exhibit high promise as therapeutic agents for the treatment of metabolic disorders, such as type II non-insulin-dependent diabetes mellitus, obesity, and hyperglycemia [3]. $\alpha$-Glucosidase inhibitors are also known to be promising as antiviral and antitumor agents that interfere with the biosynthesis of $N$-linked oligosaccharide chains [4].

Classically, efforts for the development of a new set of $\alpha$-glucosidase inhibitors have focused mainly on sugar mimics, such as disaccharides, iminosugars, carbasugars, and thiosugars. However, numerous disadvantages exist for this strategy, including poor activity, low natural abundance, or complicated stereochemistry, which makes them difficult to approach synthetically. A more modern approach is to investigate nonsugar $\alpha$-glucosidase inhibitors [5-9].

We had previously reported that 3-[4-(phenylsulfonamido)benzoyl]-2H-1-benzopyran-2-one derivatives [10], designed by incorporating the phenylsulfonamide chalcone substructure into the benzopyran backbone shown in Figure 1 [11], have the potential to act as a new class of nonsugar $\alpha$-glucosidase inhibitors. The preliminary study on the structure-activity relationship had focused on the C-ring, which showed that the modification of this substructure with halogen or bulky groups can reduce the inhibitory activity, whereas introducing diethylamino group at $\mathrm{C} 7$ and methoxy and hydroxy groups at C6 and C7, respectively, can increase the potency significantly. In addition, we found that compounds bearing the methyl group displayed higher activities than those bearing a hydrogen atom, such as compounds $\mathbf{7 u}$ and $\mathbf{7 j}$ (Figure 1). This observation 


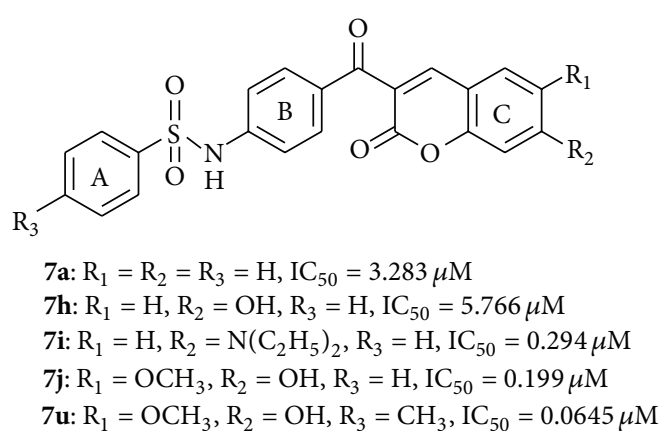

Figure 1: Chemical structures of compounds $\mathbf{7 a}, \mathbf{7 h}, \mathbf{7 i}, \mathbf{7} \mathbf{j}$, and $\mathbf{7 u}$.

encouraged us to further investigate the influence of A-ring substituents on the $\alpha$-glucosidase inhibitory activity of 3-[4-(phenylsulfonamido)benzoyl]-2H-1-benzopyran-2-one derivatives.

In the present study, a series of new substituted 3-[4(phenylsulfonamido)benzoyl]-2H-1-benzopyran-2-one derivatives (Scheme 1), bearing halogen atoms, methoxy, or tertbutyl groups at the para-position of the A-ring while keeping the favorable substituents on the C-ring unchanged, were synthesized and assayed on yeast $\alpha$-glucosidase to explore the effect of these substituents on the $\alpha$-glucosidase inhibitory activity and obtain a comprehensive understanding of the structure-activity relationships of this class of compounds.

\section{Results}

The compounds 2-9 were synthesized according to the literature [12]. 4-Nitrobenzoic acid with thionyl chloride and 1,2,3-benzotriazole was refluxed to get 4-nitrobenzoyl1,2,3-benzotriazole (2) as shown in Scheme 1 [12]. The resulting compound reacted with ethyl acetoacetate in the presence of sodium hydride, followed by hydrolysis to form ethyl 3-(4-nitrophenyl)-3-oxopropanoate (3) [13], which was subsequently reduced with stannous chloride in ethyl acetate to provide the key intermediate ethyl 3-(4aminophenyl)-3-oxopropanoate (4) as shown in Scheme 1 [14]. Afterward, compound 4 was acylated with a series of para-substituted phenylsulfonyl chlorides to obtain ethyl 3-[4-(phenylsulfonamido)phenyl]-3-oxopropanoates (5-9). Lastly, compounds 5-9 were reacted separately with substituted salicylaldehydes via Knoevenagel condensation. The target compounds 10-26 were prepared as shown in Scheme 1 and seventeen target compounds were obtained in modest yields.

All target compounds were evaluated spectrophotometrically at $490 \mathrm{~nm}$ on yeast $\alpha$-glucosidase to evaluate their potential as $\alpha$-glucosidase inhibitors. Acarbose was included as a reference compound. The in vitro $\alpha$-glucosidase inhibitory activity of the test compounds was assayed as described previously [10]. The test compounds were initially assayed for their ability to inhibit $\alpha$-glucosidase at a concentration of $1 \mu \mathrm{g} \cdot \mathrm{mL}^{-1}$. Compounds that displayed more than $50 \%$ inhibition were selected for concentration dependent activity evaluation and calculation of $\mathrm{IC}_{50}$ values, as shown in Table 1.

\section{Discussion}

Except for compound $10\left(\mathrm{IC}_{50}=7.568 \mu \mathrm{M}\right)$ in the halogen substituted series, most of the tested compounds showed stronger inhibitory activity than that of compound $7 \mathbf{a}$, previously studied in [10] $\left(\mathrm{IC}_{50}=3.283 \mu \mathrm{M}\right)$. Compound $18\left(\mathrm{IC}_{50}=0.014 \mu \mathrm{M}\right)$ presented as the strongest inhibitor which was nearly 235 times higher than compound 7a. The activities of compounds $\mathbf{1 3}\left(\mathrm{IC}_{50}=0.075 \mu \mathrm{M}\right), \mathbf{1 7}\left(\mathrm{IC}_{50}=\right.$ $0.025 \mu \mathrm{M})$, and $21\left(\mathrm{IC}_{50}=0.036 \mu \mathrm{M}\right)$ were increased about 3,8 , and 6 times, respectively, compared with the activity of compound $7 \mathbf{j}$, previously described in [10] $\left(\mathrm{IC}_{50}=0.199 \mu \mathrm{M}\right)$. This observation suggested that the halogen atoms at the para-position of the A-ring could greatly enhance the activity. However, this fact was contrary to the disadvantageous effect observed for the halogen groups presented on the C-ring [10].

The activity of compound $22\left(\mathrm{IC}_{50}=0.073 \mu \mathrm{M}\right)$ in the methoxy substituted series $\left(\mathrm{R}_{3}=\mathrm{OCH}_{3}\right)$ was 45 -fold higher than that of compound 7a. Compound 23 displayed an $\mathrm{IC}_{50}$ value of $0.018 \mu \mathrm{M}$, which was 320 -fold more potent than compound $\mathbf{7 h}$ and was ranked as the strongest inhibitor in this series.Compounds $\mathbf{2 4}\left(\mathrm{IC}_{50}=0.037 \mu \mathrm{M}\right)$ and $\mathbf{2 5}\left(\mathrm{IC}_{50}\right.$ $=0.069 \mu \mathrm{M})$ also exhibited a much better inhibitory activity compared with compounds $\mathbf{7 i}$ and $\mathbf{7 j}$. Interestingly, most compounds in this series showed higher activities than those in the methyl substituted series. The activities of compounds 22, 23, and 24 were higher than those of compounds 71 $\left(\mathrm{IC}_{50}=3.577 \mu \mathrm{M}\right), 7 \mathrm{~s}\left(\mathrm{IC}_{50}=1.125 \mu \mathrm{M}\right)$, and $7 \mathrm{t}\left(\mathrm{IC}_{50}=\right.$ $0.347 \mu \mathrm{M})$, respectively. All these observations indicated that the methoxy group substituted at the para-position of the A-ring was favorable for increasing the $\alpha$-glucosidase inhibitory activity of 3-[4-(phenylsulfonamido)benzoyl]-2H1-benzopyran-2-one derivatives.

The activity of compound $\mathbf{2 6}$ with tert-butyl group at the para-position of the A-ring disappeared, suggesting that this bulky group is unfavorable for binding with $\alpha$-glucosidase, which is in agreement with what we had found on the C-ring [10].

\section{Experimental}

4.1. Chemistry. Melting points were recorded using YRT-3 melting point apparatus. The ${ }^{1} \mathrm{H}$-NMR spectra were recorded in DMSO- $d_{6}$ on a Bruker ARX-300 spectrometer, and chemical shifts $(\delta)$ were expressed in ppm downfield from the TMS and were used as the internal standard. Coupling constant $(J)$ values were in $\mathrm{Hz}$. The IR spectra were determined as $\mathrm{KBr}$ pellets on the Bruker IFS- 55 spectrometer and were expressed in $\mathrm{cm}^{-1}$. The progress of the reactions was monitored by TLC using several solvent systems with different polarities. The TOF-HRMS spectra were recorded on a Bruker MicroTOFQ. All solvents and reagents were of analytical grade.

General Procedure for the Synthesis of Compounds 10-26. Compounds 5-9 $(1.4 \mathrm{mmol})$ reacted with substituted salicylaldehyde $(1.6 \mathrm{mmol})$ separately; five drops of piperidine, one drop of glacial acetic acid, and ethanol $(10 \mathrm{~mL})$ were added and stirred under reflux in an argon atmosphere for $0.5 \mathrm{~h}$. The mixture was cooled to room temperature, filtered 
<smiles></smiles>

$$
\begin{aligned}
& \text { 10: } \mathrm{R}_{1}=H, \mathrm{R}_{2}=H, \mathrm{R}_{3}=F \\
& \text { 11: } \mathrm{R}_{1}=H, \mathrm{R}_{2}=\mathrm{OH}, \mathrm{R}_{3}=F \\
& \text { 12: } \mathrm{R}_{1}=H, \mathrm{R}_{2}=\mathrm{N}\left(\mathrm{C}_{2} \mathrm{H}_{5}\right)_{2}, \mathrm{R}_{3}=\mathrm{F} \\
& \text { 13: } \mathrm{R}_{1}=\mathrm{OCH}_{3}, \mathrm{R}_{2}=\mathrm{OH}, \mathrm{R}_{3}=\mathrm{F} \\
& \text { 14: } \mathrm{R}_{1}=\mathrm{H}, \mathrm{R}_{2}=\mathrm{H}, \mathrm{R}_{3}=\mathrm{Cl} \\
& \text { 15: } \mathrm{R}_{1}=\mathrm{H}, \mathrm{R}_{2}=\mathrm{OH}, \mathrm{R}_{3}=\mathrm{Cl} \\
& \text { 16: } \mathrm{R}_{1}=H, \mathrm{R}_{2}=\mathrm{N}\left(\mathrm{C}_{2} \mathrm{H}_{5}\right)_{2}, \mathrm{R}_{3}=\mathrm{Cl} \\
& \text { 17: } \mathrm{R}_{1}=O \mathrm{OCH}_{3}, \mathrm{R}_{2}=\mathrm{OH}, \mathrm{R}_{3}=\mathrm{Cl} \\
& \text { 18: } \mathrm{R}_{1}=H, \mathrm{R}_{2}=H, \mathrm{R}_{3}=\mathrm{Br}
\end{aligned}
$$

$$
\begin{aligned}
& \text { 19: } \mathrm{R}_{1}=\mathrm{H}, \mathrm{R}_{2}=\mathrm{OH}, \mathrm{R}_{3}=\mathrm{Br} \\
& \text { 20: } \mathrm{R}_{1}=\mathrm{H}, \mathrm{R}_{2}=\mathrm{N}\left(\mathrm{C}_{2} \mathrm{H}_{5}\right)_{2}, \mathrm{R}_{3}=\mathrm{Br} \\
& \text { 21: } \mathrm{R}_{1}=O C \mathrm{OH}_{3}, \mathrm{R}_{2}=\mathrm{OH}, \mathrm{R}_{3}=\mathrm{Br} \\
& \text { 22: } \mathrm{R}_{1}=\mathrm{H}, \mathrm{R}_{2}=\mathrm{H}, \mathrm{R}_{3}=\mathrm{OCH}_{3} \\
& \text { 23: } \mathrm{R}_{1}=\mathrm{H}, \mathrm{R}_{2}=\mathrm{OH}, \mathrm{R}_{3}=\mathrm{OCH}_{3} \\
& \text { 24: } \mathrm{R}_{1}=\mathrm{H}, \mathrm{R}_{2}=\mathrm{N}\left(\mathrm{C}_{2} \mathrm{H}_{5}\right)_{2}, \mathrm{R}_{3}=\mathrm{OCH}_{3} \\
& \text { 25: } \mathrm{R}_{1}=O C \mathrm{OCH}_{3}, \mathrm{R}_{2}=\mathrm{OH}, \mathrm{R}_{3}=\mathrm{OCH}_{3} \\
& \text { 26: } \mathrm{R}_{1}=\mathrm{H}, \mathrm{R}_{2}=\mathrm{N}\left(\mathrm{C}_{2} \mathrm{H}_{5}\right)_{2}, \mathrm{R}_{3}=\mathrm{C}\left(\mathrm{CH}_{3}\right)_{3}
\end{aligned}
$$$$
\text { 5: } \mathrm{R}_{3}=\mathrm{F}
$$$$
\text { 6: } \mathrm{R}_{3}=\mathrm{Cl}
$$$$
\text { 7: } \mathrm{R}_{3}=\mathrm{Br}
$$$$
\text { 8: } \mathrm{R}_{3}=\mathrm{OCH}_{3}
$$$$
\text { 9: } \mathrm{R}_{3}=\mathrm{C}\left(\mathrm{CH}_{3}\right)_{3}
$$

Scheme 1: Synthesis of 3-[4-(phenylsulfonamido)benzoyl]-2H-1-benzopyran-2-one derivatives 10-26. Reagents and conditions: (a) SOCl ${ }_{2}$, 1,2,3-benzotriazole, and reflux; (b) ethyl acetoacetate, $\mathrm{NaH}$, THF, and rt; (c) $\mathrm{SnCl}_{2}$, ethyl acetate, and reflux; (d) substituted benzenesulfonyl chloride, pyridine, $\mathrm{CH}_{2} \mathrm{Cl}_{2},-10^{\circ} \mathrm{C}$, and then $\mathrm{rt}$; (e) substituted salicylaldehyde, piperidine, acetic acid, EtOH, and reflux.

TABLE 1: The $\alpha$-glucosidase inhibitory activity of new substituted 3-[4-(phenylsulfonamido)benzoyl]-2H-1-benzopyran-2-one derivatives.

\begin{tabular}{lcccc}
\hline Number & $\mathrm{R}_{1}$ & $\mathrm{R}_{2}$ & $\mathrm{R}_{3}$ & $\mathrm{IC}_{50}(\mu \mathrm{M})^{\mathrm{a}}$ \\
\hline $\mathbf{1 0}$ & $\mathrm{H}$ & $\mathrm{H}$ & $\mathrm{F}$ & $7.568 \pm 0.575$ \\
$\mathbf{1 1}$ & $\mathrm{H}$ & $\mathrm{OH}$ & $\mathrm{F}$ & $0.282 \pm 0.038$ \\
$\mathbf{1 2}$ & $\mathrm{H}$ & $\mathrm{N}\left(\mathrm{C}_{2} \mathrm{H}_{5}\right)_{2}$ & $\mathrm{~F}$ & $0.085 \pm 0.014$ \\
$\mathbf{1 3}$ & $\mathrm{OCH}_{3}$ & $\mathrm{OH}$ & $\mathrm{F}$ & $0.075 \pm 0.012$ \\
$\mathbf{1 4}$ & $\mathrm{H}$ & $\mathrm{H}$ & $\mathrm{Cl}$ & $1.088 \pm 0.232$ \\
$\mathbf{1 5}$ & $\mathrm{H}$ & $\mathrm{OH}$ & $\mathrm{Cl}$ & $0.171 \pm 0.034$ \\
$\mathbf{1 6}$ & $\mathrm{H}$ & $\mathrm{N}\left(\mathrm{C}_{2} \mathrm{H}_{5}\right)_{2}$ & $\mathrm{Cl}$ & $0.108 \pm 0.019$ \\
$\mathbf{1 7}$ & $\mathrm{OCH}$ & $\mathrm{Cr}$ & $0.025 \pm 0.005$ \\
$\mathbf{1 8}$ & $\mathrm{H}$ & $\mathrm{H}$ & $\mathrm{Br}$ & $0.014 \pm 0.003$ \\
$\mathbf{1 9}$ & $\mathrm{H}$ & $\mathrm{OH}$ & $\mathrm{Br}$ & $0.199 \pm 0.016$ \\
$\mathbf{2 0}$ & $\mathrm{H}$ & $\mathrm{N}\left(\mathrm{C}_{2} \mathrm{H}_{5}\right)_{2}$ & $0.125 \pm 0.035$ \\
$\mathbf{2 1}$ & $\mathrm{OH}$ & $\mathrm{Br}$ & $0.036 \pm 0.008$ \\
$\mathbf{2 2}$ & $\mathrm{OCH}$ & $\mathrm{H}$ & $\mathrm{OCH}_{3}$ & $0.073 \pm 0.012$ \\
$\mathbf{2 3}$ & $\mathrm{H}$ & $\mathrm{OH}$ & $\mathrm{OCH}_{3}$ & $0.018 \pm 0.003$ \\
$\mathbf{2 4}$ & $\mathrm{H}$ & $\mathrm{OCH}_{3}$ & $0.037 \pm 0.006$ \\
$\mathbf{2 5}$ & $\mathrm{H}$ & $\mathrm{N}\left(\mathrm{C}_{2} \mathrm{H}_{5}\right)_{2}$ & $\mathrm{OCH}_{3}$ & $0.069 \pm 0.011$ \\
$\mathbf{2 6}$ & $\mathrm{OCH}$ & $\mathrm{OH}$ & $\mathrm{CH}_{3}$ & $-\mathrm{CH}_{3}$ \\
Acarbose & $\mathrm{H}$ & $\mathrm{N}\left(\mathrm{C}_{2} \mathrm{H}_{5}\right)_{2}$ & $29.26 \pm 3.23$ \\
\hline
\end{tabular}

${ }^{\mathrm{a}} \mathrm{IC}_{50}$ values are represented as means $\pm \mathrm{SD}(n=2-3) .{ }^{\mathrm{b}}$ Less than $50 \%$ inhibition at $10 \mu \mathrm{g} / \mathrm{mL}$.

to collect the solid (if there was no precipitate, $25 \mathrm{~mL}$ water was added), and then washed with a small amount of ethanol. In most cases, the products were sufficiently pure. Products with impurities were purified via column chromatography, petroleum ether, and ethyl acetate systems with different polarities as eluent.
4.1.1. 3-[4-(4-Fluorophenylsulfonamido])benzoyl]-2H-1- benzopyran-2-one (10). White solid, 49\% yield. M.p.: 204.5$205.5^{\circ} \mathrm{C} ;{ }^{1} \mathrm{H}-\mathrm{NMR}$ (DMSO- $\left.d_{6}, \mathrm{ppm}\right): \delta 7.22(\mathrm{~d}, 2 \mathrm{H}, J=8.7 \mathrm{~Hz}$, $\left.\mathrm{H}-3^{\prime}, 5^{\prime}\right), 7.44$ (m, 4H, H-6, 8, 3', $\left.5^{\prime \prime}\right), 7.73$ (m, 1H, H-7), 7.83 (m, 3H, H-5, $\left.2^{\prime}, 6^{\prime}\right), 7.90$ (m, 2H, H-2" , 6" $), 8.34$ (s, 1H, H-4), 11.03 (s, 1H, NH); IR (KBr, cm ${ }^{-1}$ ): 3432, 3240, 1732, 1652, 1601, 
1567, 1510, 1240, 1169, 838, 800; TOF-HRMS: $m / z 424.0649$ $[\mathrm{M}+\mathrm{H}]^{+}\left(\mathrm{C}_{22} \mathrm{H}_{15} \mathrm{FNO}_{5} \mathrm{~S}\right.$ requires 424.0656).

4.1.2.3-[4-(4-Fluorophenylsulfonamido)benzoyl]-7-hydroxy2H-1-benzopyran-2-one (11). Brown solid, 54\% yield. M.p.: 159-160 ${ }^{\circ} \mathrm{C} ;{ }^{1} \mathrm{H}-\mathrm{NMR}$ (DMSO- $\left.d_{6}, \mathrm{ppm}\right): \delta 6.76$ (s, $\left.1 \mathrm{H}, \mathrm{H}-8\right)$, $6.82(\mathrm{~d}, 1 \mathrm{H}, J=8.6 \mathrm{~Hz}, \mathrm{H}-6), 7.12\left(\mathrm{~d}, 2 \mathrm{H}, J=8.6 \mathrm{~Hz}, \mathrm{H}-3^{\prime}, 5^{\prime}\right)$, $7.39\left(\mathrm{t}, 2 \mathrm{H}, J=8.8 \mathrm{~Hz}, \mathrm{H}-3^{\prime \prime}, 5^{\prime \prime}\right), 7.64(\mathrm{~d}, 1 \mathrm{H}, J=8.6 \mathrm{~Hz}, \mathrm{H}-5)$, $7.70\left(\mathrm{~d}, 2 \mathrm{H}, J=8.6 \mathrm{~Hz}, \mathrm{H}-2^{\prime \prime}, 6^{\prime \prime}\right), 7.88(\mathrm{dd}, 2 \mathrm{H}, J=8.8 \mathrm{~Hz}$ and $\left.5.2 \mathrm{~Hz}, \mathrm{H}-2^{\prime}, 6^{\prime}\right), 8.22$ (s, 1H, H-4); IR $\left(\mathrm{KBr}, \mathrm{cm}^{-1}\right): 3474,2924$, 1730, 1601, 1228, 1166, 839, 816; TOF-HRMS: $m / z 440.0599$ $[\mathrm{M}+\mathrm{H}]^{+}\left(\mathrm{C}_{22} \mathrm{H}_{15} \mathrm{FNO}_{6} \mathrm{~S}\right.$ requires 440.0605).

4.1.3. 3-[4-(4-Fluorophenylsulfonamido)benzoyl]-7-(N,N-diethylamino)-2H-1-benzopyran-2-one (12). Yellow solid, 51\% yield. M.p.: $207-208^{\circ} \mathrm{C}^{1}{ }^{1} \mathrm{H}-\mathrm{NMR}$ (DMSO- $d_{6}, \mathrm{ppm}$ ): $\delta 1.13$ $\left(\mathrm{t}, 6 \mathrm{H}, J=6.9 \mathrm{~Hz}, 2 \mathrm{CH}_{2} \mathrm{CH}_{3}\right), 3.48(\mathrm{q}, 4 \mathrm{H}, J=6.9 \mathrm{~Hz}$, $\left.2 \mathrm{CH}_{2} \mathrm{CH}_{3}\right), 6.57$ (s, $\left.1 \mathrm{H}, \mathrm{H}-8\right), 6.75(\mathrm{~d}, 1 \mathrm{H}, J=9.0 \mathrm{~Hz}, \mathrm{H}-6)$, $7.18\left(\mathrm{~d}, 2 \mathrm{H}, J=8.6 \mathrm{~Hz}, \mathrm{H}-3^{\prime}, 5^{\prime}\right), 7.43\left(\mathrm{t}, 2 \mathrm{H}, J=8.7 \mathrm{~Hz}, \mathrm{H}-3^{\prime \prime}\right.$, $\left.5^{\prime \prime}\right), 7.56(\mathrm{~d}, 1 \mathrm{H}, J=9.0 \mathrm{~Hz}, \mathrm{H}-5), 7.68(\mathrm{~d}, 2 \mathrm{H}, J=8.6 \mathrm{~Hz}, \mathrm{H}-$ $\left.2^{\prime}, 6^{\prime}\right), 7.92\left(\mathrm{dd}, 2 \mathrm{H}, J=8.7 \mathrm{~Hz}\right.$ and $\left.5.3 \mathrm{~Hz}, \mathrm{H}-2^{\prime \prime}, 6^{\prime \prime}\right), 8.17$ (s, $1 \mathrm{H}, \mathrm{H}-4), 10.90$ (s, 1H, NH); IR (KBr, cm $\left.{ }^{-1}\right): 3441,3164,2973$, 1690, 1617, 1599, 1580, 1508, 1234, 1166, 827, 812; TOF-HRMS: $m / z 495.1384[\mathrm{M}+\mathrm{H}]^{+}\left(\mathrm{C}_{26} \mathrm{H}_{24} \mathrm{FN}_{2} \mathrm{O}_{5} \mathrm{~S}\right.$ requires 495.1391).

4.1.4. 3-[4-(4-Fluorophenylsulfonamido)benzoyl]-7-hydroxy6-methoxy-2H-1-benzopyran-2-one (13). Yellow solid, 39\% yield. M.p.: $226-227^{\circ} \mathrm{C} ;{ }^{1} \mathrm{H}-\mathrm{NMR}$ (DMSO- $\left.d_{6}, \mathrm{ppm}\right): \delta 3.81$ (s, $\left.3 \mathrm{H}, \mathrm{OCH}_{3}\right), 6.84$ (s, $\left.1 \mathrm{H}, \mathrm{H}-8\right), 7.22\left(\mathrm{~d}, 2 \mathrm{H}, J=8.5 \mathrm{~Hz}, \mathrm{H}-3^{\prime}\right.$, $5^{\prime}$ ), 7.35 (s, 1H, H-5), 7.44 (m, 2H, H-3", $\left.5^{\prime \prime}\right), 7.77$ (d, 2H, J $\left.=8.6 \mathrm{~Hz}, \mathrm{H}-2^{\prime}, 6^{\prime}\right), 7.91\left(\mathrm{~m}, 2 \mathrm{H}, \mathrm{H}-2^{\prime \prime}, 6^{\prime \prime}\right), 8.22(\mathrm{~s}, 1 \mathrm{H}, \mathrm{H}-$ 4), 10.89 (s, $1 \mathrm{H}, \mathrm{NH})$; IR (KBr, cm $\left.{ }^{-1}\right): 3429,3206,2922,1680$, 1658, 1602, 1569, 1509, 1261, 1156, 840, 790; TOF-HRMS: $m / z$ 470.0704 $[\mathrm{M}+\mathrm{H}]^{+}\left(\mathrm{C}_{23} \mathrm{H}_{17} \mathrm{FNO}_{7} \mathrm{~S}\right.$ requires 470.0711).

4.1.5. 3-[4-(4-Chlorophenylsulfonamido)benzoyl]-2H-1-benzopyran-2-one (14). White solid, $87 \%$ yield. M.p.: $208-209^{\circ} \mathrm{C}$; ${ }^{1} \mathrm{H}-\mathrm{NMR}$ (DMSO- $\left.d_{6}, \mathrm{ppm}\right): \delta 7.21\left(\mathrm{~d}, 2 \mathrm{H}, J=8.7 \mathrm{~Hz}, \mathrm{H}-3^{\prime}\right.$, $5^{\prime}$ ), 7.42 (m, 2H, H-6, 8), 7.66 (d, $\left.2 \mathrm{H}, J=8.7 \mathrm{~Hz}, \mathrm{H}-3^{\prime \prime}, 5^{\prime \prime}\right)$, 7.73 (m, 1H, H-7), 7.84 (m, 5H, H-5, 2', 6' $\left., 2^{\prime \prime}, 6^{\prime \prime}\right), 8.34$ (s, 1H, $\mathrm{H}-4), 11.08$ (s, 1H, NH); IR (KBr, cm $\left.{ }^{-1}\right): 3441,3243,1736,1653$, 1603, 1510, 1240, 1164, 828, 801; TOF-HRMS: $m / z 440.0354$ $[\mathrm{M}+\mathrm{H}]^{+}\left(\mathrm{C}_{22} \mathrm{H}_{15} \mathrm{ClNO}_{5} \mathrm{~S}\right.$ requires 440.0360).

4.1.6. 3-[4-(4-Chlorophenylsulfonamido)benzoyl]-7-hydroxy2H-1-benzopyran-2-one (15). Brown solid, 24\% yield. M.p.: 186-187 ${ }^{\circ} \mathrm{C} ;{ }^{1} \mathrm{H}-\mathrm{NMR}$ (DMSO- $d_{6}, \mathrm{ppm}$ ): $\delta 6.75$ (s, $1 \mathrm{H}, \mathrm{H}-8$ ), $6.81(\mathrm{~d}, 1 \mathrm{H}, J=8.6 \mathrm{~Hz}, \mathrm{H}-6), 7.01$ (d, $2 \mathrm{H}, J=8.6 \mathrm{~Hz}, \mathrm{H}-3^{\prime}$, $\left.5^{\prime}\right), 7.55\left(\mathrm{~d}, 2 \mathrm{H}, J=8.5 \mathrm{~Hz}, \mathrm{H}-3^{\prime \prime}, 5^{\prime \prime}\right), 7.62(\mathrm{~d}, 3 \mathrm{H}, J=8.5 \mathrm{~Hz}$, $\left.\mathrm{H}-5,2^{\prime}, 6^{\prime}\right), 7.77$ (d, 2H, $\left.J=8.5 \mathrm{~Hz}, \mathrm{H}-2^{\prime \prime}, 6^{\prime \prime}\right), 8.16$ (s, $1 \mathrm{H}$, $\mathrm{H}-4)$; IR (KBr, cm ${ }^{-1}$ ): 3445, 1716, 1601, 1506, 1225, 1162, 850, 790; TOF-HRMS: $m / z 456.0303[\mathrm{M}+\mathrm{H}]^{+}\left(\mathrm{C}_{22} \mathrm{H}_{15} \mathrm{ClNO}_{6} \mathrm{~S}\right.$ requires 456.0309 ).

4.1.7. 3-(4-Chlorophenylsulfonamidobenzoyl)-7-(N,N-diethylamino)-2H-1-benzopyran-2-one (16). Brown solid, 31\% yield. M.p.: $>250{ }^{\circ} \mathrm{C} ;{ }^{1} \mathrm{H}-\mathrm{NMR}\left(\mathrm{DMSO}-d_{6}, \mathrm{ppm}\right): \delta 1.13(\mathrm{t}, 6 \mathrm{H}$,
$\left.J=7.0 \mathrm{~Hz}, 2 \mathrm{CH}_{3}\right), 3.47\left(\mathrm{q}, 4 \mathrm{H}, J=7.0 \mathrm{~Hz}, 2 \mathrm{CH}_{2}\right), 6.56(\mathrm{~s}, 1 \mathrm{H}$, $\mathrm{H}-8), 6.76$ (d, $1 \mathrm{H}, J=8.6 \mathrm{~Hz}, \mathrm{H}-6), 7.09$ (d, 2H, $J=8.2 \mathrm{~Hz}, \mathrm{H}-$ $\left.3^{\prime}, 5^{\prime}\right), 7.61\left(\mathrm{~m}, 5 \mathrm{H}, \mathrm{H}-5,2^{\prime}, 6^{\prime}, 3^{\prime \prime}, 5^{\prime \prime}\right), 7.82(\mathrm{~d}, 2 \mathrm{H}, J=8.1 \mathrm{~Hz}$, $\left.\mathrm{H}-2^{\prime \prime}, 6^{\prime \prime}\right), 8.11$ (s, $\left.1 \mathrm{H}, \mathrm{H}-4\right)$; IR $\left(\mathrm{KBr}, \mathrm{cm}^{-1}\right)$ : 3433, 3173, 2973, 1718, 1689, 1617, 1581, 1508, 1233, 1163, 825, 789; TOF-HRMS: $m / z 511.1089[\mathrm{M}+\mathrm{H}]^{+}\left(\mathrm{C}_{26} \mathrm{H}_{24} \mathrm{ClN}_{2} \mathrm{O}_{5} \mathrm{~S}\right.$ requires 511.1095).

4.1.8. 3-[4-(4-Chlorophenylsulfonamido)benzoyl]-7-hydroxy 6 -methoxy-2H-1-benzopyran-2-one (17). Yellow solid, 37\% yield. M.p.: $>250^{\circ} \mathrm{C}$; ${ }^{1} \mathrm{H}-\mathrm{NMR}$ (DMSO- $\left.d_{6}, \mathrm{ppm}\right): \delta 3.81$ (s, $3 \mathrm{H}, \mathrm{OCH}_{3}$ ), 6.84 (s, $\left.1 \mathrm{H}, \mathrm{H}-5\right), 7.22\left(\mathrm{~d}, 2 \mathrm{H}, \mathrm{J}=8.4 \mathrm{~Hz}, \mathrm{H}-3^{\prime}\right.$, $\left.5^{\prime}\right), 7.35$ (s, $\left.1 \mathrm{H}, \mathrm{H}-8\right), 7.66$ (d, $\left.2 \mathrm{H}, J=8.4 \mathrm{~Hz}, \mathrm{H}-3^{\prime \prime}, 5^{\prime \prime}\right), 7.77$ $\left(\mathrm{d}, 2 \mathrm{H}, J=8.5 \mathrm{~Hz}, \mathrm{H}-2^{\prime}, 6^{\prime}\right), 7.84\left(\mathrm{~d}, 2 \mathrm{H}, J=8.5 \mathrm{~Hz}, \mathrm{H}-2^{\prime \prime}, 6^{\prime \prime}\right)$, 8.22 (s, 1H, H-4), 10.91 (s, 1H, NH); IR (KBr, cm ${ }^{-1}$ ): 3433, 3310, 1681, 1601, 1569, 1509, 1258, 1160, 847, 827; TOF-HRMS: $m / z$ $508.0228[\mathrm{M}+\mathrm{Na}]^{+}\left(\mathrm{C}_{23} \mathrm{H}_{16} \mathrm{ClNNaO}_{7} \mathrm{~S}\right.$ requires 508.0234).

4.1.9. 3-[4-(4-Bromophenylsulfonamido) benzoyl]-2H-1-benzopyran-2-one (18). Yellow solid, 27\% yield. M.p.: $205-207^{\circ} \mathrm{C}$; ${ }^{1} \mathrm{H}-\mathrm{NMR}$ (DMSO- $\left.d_{6}, \mathrm{ppm}\right): \delta 7.20(\mathrm{~d}, 2 \mathrm{H}, J=8.7 \mathrm{~Hz}, \mathrm{H}-$ $\left.3^{\prime}, 5^{\prime}\right), 7.42$ (m, 3H, H-6, 7, 8), 7.73 (d, 1H, J = 8.6 Hz, H-5), 7.78 (m, 6H, H-2', 6', $\left.2^{\prime \prime}, 3^{\prime \prime}, 5^{\prime \prime}, 6^{\prime \prime}\right), 8.34$ (s, 1H, H-4); IR $\left(\mathrm{KBr}, \mathrm{cm}^{-1}\right)$ : 3433, 3245, 1721, 1604, 1571, 1510, 1240, 1163, 823, 758; TOF-HRMS: $m / z 483.9849[\mathrm{M}+\mathrm{H}]^{+}\left(\mathrm{C}_{22} \mathrm{H}_{15} \mathrm{BrNO}_{5} \mathrm{~S}\right.$ requires 483.9855$)$.

4.1.10. 3-[4-(4-Bromophenylsulfonamido)benzoyl]-7-hydroxy2H-1-benzopyran-2-one (19). Yellow solid, 43\% yield. M.p.: 203.5-204.5 ${ }^{\circ}$; ${ }^{1} \mathrm{H}-\mathrm{NMR}$ (DMSO- $d_{6}, \mathrm{ppm}$ ): $\delta 6.77$ (s, $1 \mathrm{H}, \mathrm{H}-$ 8), $6.83(\mathrm{~d}, 1 \mathrm{H}, J=8.6 \mathrm{~Hz}, \mathrm{H}-6), 7.22\left(\mathrm{~d}, 2 \mathrm{H}, J=8.7 \mathrm{~Hz}, \mathrm{H}-3^{\prime}\right.$, $\left.5^{\prime}\right), 7.66(\mathrm{~d}, 1 \mathrm{H}, J=8.6 \mathrm{~Hz}, \mathrm{H}-5), 7.76\left(\mathrm{~m}, 6 \mathrm{H}, \mathrm{H}-2^{\prime}, 6^{\prime}, 2^{\prime \prime}, 3^{\prime \prime}\right.$, $\left.5^{\prime \prime}, 6^{\prime \prime}\right), 8.26$ (s, 1H, H-4), $10.94(\mathrm{~s}, 1 \mathrm{H}, \mathrm{OH}), 11.03$ (s, 1H, NH); IR $\left(\mathrm{KBr}, \mathrm{cm}^{-1}\right): 3205,1715,1601,1573,1508,1226,1161,852$, 805; TOF-HRMS: $m / z 499.9798[\mathrm{M}+\mathrm{H}]^{+}\left(\mathrm{C}_{22} \mathrm{H}_{15} \mathrm{BrNO}_{6} \mathrm{~S}\right.$ requires 499.9804$)$.

4.1.11. 3-[4-(4-Bromophenylsulfonamido)benzoyl]-7-(N,N -diethylamino)-2H-1-benzopyran-2-one (20). Yellow solid, 50\% yield. M.p.: $215-216^{\circ} \mathrm{C}$; ${ }^{1} \mathrm{H}-\mathrm{NMR}$ (DMSO- $d_{6}, \mathrm{ppm}$ ): $\delta 1.14$ $\left(\mathrm{t}, 6 \mathrm{H}, J=6.9 \mathrm{~Hz}, 2 \mathrm{CH}_{2} \mathrm{CH}_{3}\right), 3.47(\mathrm{q}, 4 \mathrm{H}, J=6.9 \mathrm{~Hz}$, $\left.2 \mathrm{CH}_{2} \mathrm{CH}_{3}\right), 6.58(\mathrm{~s}, 1 \mathrm{H}, \mathrm{H}-8), 6.76(\mathrm{~d}, 1 \mathrm{H}, J=8.9 \mathrm{~Hz}$, $\mathrm{H}-6), 7.20$ (d, 2H, J = 8.7 Hz, H-3', 5'), 7.59 (d, 1H, $J=9.0 \mathrm{~Hz}, \mathrm{H}-5), 7.69\left(\mathrm{~d}, 2 \mathrm{H}, J=8.7 \mathrm{~Hz}, \mathrm{H}-3^{\prime \prime}, 5^{\prime \prime}\right)$, 7.77 (q, 4H, H-2', 6', 2"' $\left.6^{\prime \prime}\right), 8.17$ (s, 1H, H-4), 10.95 $(\mathrm{s}, 1 \mathrm{H}, \mathrm{NH})$; IR $\left(\mathrm{KBr}, \mathrm{cm}^{-1}\right): 3438,3180,2974,1715$, $1688,1617,1600,1579,1508,1233,1162,823,740$; TOFHRMS: $m / z 555.0584[\mathrm{M}+\mathrm{H}]^{+}\left(\mathrm{C}_{26} \mathrm{H}_{24} \mathrm{BrN}_{2} \mathrm{O}_{5} \mathrm{~S}\right.$ requires $555.0590)$.

4.1.12. 3-[4-(4-Bromophenylsulfonamido)benzoyl]-7-hydroxy6-methoxy-2H-1-benzopyran-2-one (21). Yellow solid, 33\% yield. M.p.: $>250{ }^{\circ} \mathrm{C}$; ${ }^{1} \mathrm{H}-\mathrm{NMR}$ (DMSO- $\left.d_{6}, \mathrm{ppm}\right): \delta 3.81$ (s, $\left.3 \mathrm{H}, \mathrm{OCH}_{3}\right), 6.84(\mathrm{~s}, 1 \mathrm{H}, \mathrm{H}-5), 7.21\left(\mathrm{~d}, 2 \mathrm{H}, J=8.6 \mathrm{~Hz}, \mathrm{H}-3^{\prime}, 5^{\prime}\right)$, 7.36 (s, 1H, H-8), 7.77 (m, 6H, H-2', $\left.6^{\prime}, 2^{\prime \prime}, 3^{\prime \prime}, 5^{\prime \prime}, 6^{\prime \prime}\right), 8.22(\mathrm{~s}$, $1 \mathrm{H}, \mathrm{H}-4), 10.93$ (s, $1 \mathrm{H}, \mathrm{NH})$; IR (KBr, cm $\left.{ }^{-1}\right)$ : 3426, 3304, 2924, 1682, 1602, 1569, 1510, 1257, 1157, 823, 787; TOF-HRMS: $m / z$ $529.9904[\mathrm{M}+\mathrm{H}]^{+}\left(\mathrm{C}_{23} \mathrm{H}_{17} \mathrm{BrNO}_{7} \mathrm{~S}\right.$ requires 529.9910). 
4.1.13. 3-[4-(4-Methoxyphenylsulfonamido)benzoyl]-2H-1-benzopyran-2-one (22). White solid, 58\% yield. M.p.: 175.5$176.5^{\circ} \mathrm{C} ;{ }^{1} \mathrm{H}-\mathrm{NMR}$ (DMSO- $\left.d_{6}, \mathrm{ppm}\right): \delta 3.81\left(\mathrm{~s}, 3 \mathrm{H}, \mathrm{OCH}_{3}\right.$ ), $7.11\left(\mathrm{~d}, 2 \mathrm{H}, J=8.9 \mathrm{~Hz}, \mathrm{H}-3^{\prime \prime}, 5^{\prime \prime}\right), 7.20\left(\mathrm{~d}, 2 \mathrm{H}, J=8.7 \mathrm{~Hz}, \mathrm{H}-3^{\prime}\right.$, $\left.5^{\prime}\right), 7.44(\mathrm{~m}, 2 \mathrm{H}, \mathrm{H}-6,8), 7.70(\mathrm{~d}, 1 \mathrm{H}, J=8.5 \mathrm{~Hz}, \mathrm{H}-7), 7.78$ (m, $\left.5 \mathrm{H}, \mathrm{H}-5,2^{\prime}, 6^{\prime}, 2^{\prime \prime}, 6^{\prime \prime}\right), 8.33$ (s, 1H, H-4), 10.88 (s, 1H, NH); IR $\left(\mathrm{KBr}, \mathrm{cm}^{-1}\right): 3444,3249,1741,1653,1598,1498,1241,1156$, 836, 801; TOF-HRMS: $m / z 436.0849[\mathrm{M}+\mathrm{H}]^{+}\left(\mathrm{C}_{23} \mathrm{H}_{18} \mathrm{NO}_{6} \mathrm{~S}\right.$ requires 436.0856 ).

4.1.14. 3-[4-(4-Methoxyphenylsulfonamido)benzoyl]-7-hydroxy-2H-1-benzopyran-2-one (23). Orange solid, 59\% yield. M.p.: $135-145^{\circ} \mathrm{C} ;{ }^{1} \mathrm{H}-\mathrm{NMR}$ (DMSO- $\left.d_{6}, \mathrm{ppm}\right): \delta 3.80$ (s, $3 \mathrm{H}$, $\left.\mathrm{OCH}_{3}\right), 6.76$ (s, $\left.1 \mathrm{H}, \mathrm{H}-8\right), 6.83(\mathrm{~d}, 1 \mathrm{H}, J=8.6 \mathrm{~Hz}, \mathrm{H}-6), 7.10$ $\left(\mathrm{d}, 2 \mathrm{H}, J=8.9 \mathrm{~Hz}, \mathrm{H}-3^{\prime \prime}, 5^{\prime \prime}\right), 7.17\left(\mathrm{~d}, 2 \mathrm{H}, J=8.7 \mathrm{~Hz}, \mathrm{H}-3^{\prime}, 5^{\prime}\right)$, $7.64(\mathrm{~d}, 1 \mathrm{H}, J=8.6 \mathrm{~Hz}, \mathrm{H}-5), 7.73\left(\mathrm{~m}, 4 \mathrm{H}, \mathrm{H}-2^{\prime}, 6^{\prime}, 2^{\prime \prime}, 6^{\prime \prime}\right)$, $8.23(\mathrm{~s}, 1 \mathrm{H}, \mathrm{H}-4)$; IR $\left(\mathrm{KBr}, \mathrm{cm}^{-1}\right): 3317,3227,1732,1646,1600$, 1497, 1228, 1146, 850, 829; TOF-HRMS: $m / z 452.0798[\mathrm{M}+\mathrm{H}]^{+}$ $\left(\mathrm{C}_{23} \mathrm{H}_{18} \mathrm{NO}_{7} \mathrm{~S}\right.$ requires 452.0805).

4.1.15. 3-[4-(4-Methoxyphenylsulfonamido)benzoyl]-7-(N,Ndiethylamino)-2H-1-benzopyran-2-one (24). Yellow solid, $37 \%$ yield. M.p.: $161-162^{\circ} \mathrm{C} ;{ }^{1} \mathrm{H}-\mathrm{NMR}$ (DMSO- $d_{6}, \mathrm{ppm}$ ): $\delta$ $1.13\left(\mathrm{~s}, 6 \mathrm{H}, J=6.9 \mathrm{~Hz}, 2 \mathrm{CH}_{3}\right), 3.47\left(\mathrm{~s}, 4 \mathrm{H}, J=6.9 \mathrm{~Hz}, 2 \mathrm{CH}_{2}\right)$, $3.80\left(\mathrm{~s}, 3 \mathrm{H}, \mathrm{OCH}_{3}\right), 6.57$ (s, $\left.1 \mathrm{H}, \mathrm{H}-8\right), 6.76$ (s, $\left.1 \mathrm{H}, \mathrm{H}-6\right), 7.08$ $\left(\mathrm{d}, 2 \mathrm{H}, J=7.6 \mathrm{~Hz}, \mathrm{H}-3^{\prime \prime}, 5^{\prime \prime}\right), 7.20\left(\mathrm{~d}, 2 \mathrm{H}, J=8.4 \mathrm{~Hz}, \mathrm{H}-3^{\prime}, 5^{\prime}\right)$, $7.58(\mathrm{~d}, 1 \mathrm{H}, J=8.7 \mathrm{~Hz}, \mathrm{H}-5), 7.70\left(\mathrm{~d}, 2 \mathrm{H}, J=8.1 \mathrm{~Hz}, \mathrm{H}-2^{\prime}, 6^{\prime}\right)$, $7.78\left(\mathrm{~d}, 2 \mathrm{H}, J=8.4 \mathrm{~Hz}, \mathrm{H}-2^{\prime \prime}, 6^{\prime \prime}\right), 8.16(\mathrm{~s}, 1 \mathrm{H}, \mathrm{H}-4), 10.76(\mathrm{~s}$, $1 \mathrm{H}, \mathrm{NH})$; IR $\left(\mathrm{KBr}, \mathrm{cm}^{-1}\right)$ : 3438, 3232, 2973, 1696, 1618, 1598, 1581, 1510, 1233, 1158, 832, 803; TOF-HRMS: $m / z 507.1584$ $[\mathrm{M}+\mathrm{H}]^{+}\left(\mathrm{C}_{27} \mathrm{H}_{27} \mathrm{~N}_{2} \mathrm{O}_{6} \mathrm{~S}\right.$ requires 507.1591).

4.1.16. 3-[4-(4-Methoxylphenylsulfonamido)benzoyl]-7-hydroxy-6-methoxy-2H-1-benzopyran-2-one (25). Yellow solid, $36 \%$ yield. M.p.: $167-168^{\circ} \mathrm{C}$; ${ }^{1} \mathrm{H}-\mathrm{NMR}$ (DMSO- $d_{6}, \mathrm{ppm}$ ): $\delta$ $3.80\left(\mathrm{~s}, 6 \mathrm{H}, 2 \mathrm{OCH}_{3}\right), 6.84(\mathrm{~s}, 1 \mathrm{H}, \mathrm{H}-5), 7.11(\mathrm{~d}, 2 \mathrm{H}, J=8.9 \mathrm{~Hz}$, $\left.\mathrm{H}-3^{\prime \prime}, 5^{\prime \prime}\right), 7.21$ (d, $\left.2 \mathrm{H}, J=8.5 \mathrm{~Hz}, \mathrm{H}-3^{\prime}, 5^{\prime}\right), 7.35$ (s, $\left.1 \mathrm{H}, \mathrm{H}-8\right)$, 7.75 (m, 4H, H-2', 6', 2", $6^{\prime \prime}$ ), 8.20 (s, 1H, H-4), 10.81 (s, $1 \mathrm{H}$, $\mathrm{NH})$; IR $\left(\mathrm{KBr}, \mathrm{cm}^{-1}\right): 3430,1715,1598,1567,1508,1263,1156$, 836, 789; TOF-HRMS: $m / z 482.0904[\mathrm{M}+\mathrm{H}]^{+}\left(\mathrm{C}_{24} \mathrm{H}_{20} \mathrm{NO}_{8} \mathrm{~S}\right.$ requires 482.0910).

4.1.17. 3-[4-(4-tert-Butylphenylsulfonamido)benzoyl]-7-(N,Ndiethylamino)-2H-1-benzopyran-2-one (26). Orange solid, $33 \%$ yield. M.p.: $118-120^{\circ} \mathrm{C} ;{ }^{1} \mathrm{H}-\mathrm{NMR}$ (DMSO- $\left.d_{6}, \mathrm{ppm}\right): \delta 1.14$ $\left(\mathrm{t}, 6 \mathrm{H}, J=7.0 \mathrm{~Hz}, 2 \mathrm{CH}_{3}\right), 1.16\left(\mathrm{~s}, 9 \mathrm{H},-\mathrm{C}\left(\mathrm{CH}_{3}\right)_{3}\right), 3.46(\mathrm{q}, 4 \mathrm{H}$, $\left.J=7.0 \mathrm{~Hz}, 2 \mathrm{CH}_{2}\right), 6.57(\mathrm{~s}, 1 \mathrm{H}, \mathrm{H}-8), 6.75(\mathrm{~d}, 1 \mathrm{H}, J=9.0 \mathrm{~Hz}, \mathrm{H}-$ 6), $7.18\left(\mathrm{~d}, 2 \mathrm{H}, J=8.7 \mathrm{~Hz}, \mathrm{H}-3^{\prime}, 5^{\prime}\right), 7.58\left(\mathrm{~m}, 3 \mathrm{H}, \mathrm{H}-5,2^{\prime}, 6^{\prime}\right)$, $7.68\left(\mathrm{~d}, 2 \mathrm{H}, J=8.7 \mathrm{~Hz}, \mathrm{H}-3^{\prime \prime}, 5^{\prime \prime}\right), 7.78\left(\mathrm{~d}, 2 \mathrm{H}, J=8.6 \mathrm{~Hz}, \mathrm{H}-2^{\prime \prime}\right.$, $\left.6^{\prime \prime}\right), 8.16(\mathrm{~s}, 1 \mathrm{H}, \mathrm{H}-4), 10.88(\mathrm{~s}, 1 \mathrm{H}, \mathrm{NH}) ; \mathrm{IR}\left(\mathrm{KBr}, \mathrm{cm}^{-1}\right): 3436$, 2967, 1721, 1618, 1600, 1510, 1234, 1162, 828, 788; TOF-HRMS: $m / z 533.2105[\mathrm{M}+\mathrm{H}]^{+}\left(\mathrm{C}_{30} \mathrm{H}_{33} \mathrm{~N}_{2} \mathrm{O}_{5} \mathrm{~S}\right.$ requires 533.2111).

4.2. Assay of the In Vitro $\alpha$-Glucosidase Inhibitory Activity. A $100 \mu \mathrm{L}$ reaction system containing $0.02 \mathrm{U}$ of $\alpha$-glucosidase, $67 \mathrm{nM}$ sodium phosphate buffer ( $\mathrm{pH} 6.8$ ), and a test compound were preincubated at $37^{\circ} \mathrm{C}$ for $10 \mathrm{~min}$. A negative control in the absence of a test compound and a blank control in the absence of either an enzyme or the test compound were run simultaneously. The reaction was initiated by the addition of $0.1 \mathrm{M}$ maltose, and the reaction mixture was incubated at room temperature for $10 \mathrm{~min}$. Then, the $200 \mu \mathrm{L}$ glucosedetecting agent was added, and the absorbance (A) at $490 \mathrm{~nm}$ was recorded on a SPECTRAmax Plus 384 reader (MD, USA). The test compounds were initially assayed for their inhibition of $\alpha$-glucosidase at a concentration of $10 \mu \mathrm{g} / \mathrm{mL}$. Calculate inhibition ratio according to $\mathrm{OD}$ value; inhibition ratio $=\left[1-\left(\mathrm{OD}_{\text {sample }}-\mathrm{OD}_{\text {blank }}\right) /\left(\mathrm{OD}_{\text {negative }}-\mathrm{OD}_{\text {blank }}\right)\right] \times$ $100 \%$. Each monoconcentration had two replicates when preliminary screened. If an inhibition ratio of more than $70 \%$ was observed, diluted 10 times and re-screened. If an inhibition ratio of re-screening was more than $70 \%$, measured $\mathrm{IC}_{50}$ value. The active compounds were consequently tested at six gradient dilute concentrations, with each concentration having two replicates. On the basis of inhibition ratio, the $\mathrm{IC}_{50}$ values were calculated using 4-Parameter Logistic Model of Xlfit software.

\section{Conflict of Interests}

The authors declare that there is no conflict of interests regarding the publication of this paper.

\section{Acknowledgment}

This work was financially supported by Key Project for Drug Innovation of the Ministry of Science and Technology of China (2009ZX09301-012).

\section{References}

[1] S. Fatmawati, K. Shimizu, and R. Kondo, "Ganoderol B: a potent $\alpha$-glucosidase inhibitor isolated from the fruiting body of Ganoderma lucidum," Phytomedicine, vol. 18, no. 12, pp.10531055, 2011.

[2] S. Bisht, R. Kant, and V. Kumar, " $\alpha$-d-Glucosidase inhibitory activity of polysaccharide isolated from Acacia tortilis gum exudate," International Journal of Biological Macromolecules, vol. 59, pp. 214-220, 2013.

[3] S. D. Kim, " $\alpha$-Glucosidase inhibitor from Buthus martensi Karsch," Food Chemistry, vol. 136, pp. 297-300, 2013.

[4] W. Hakamata, M. Kurihara, H. Okuda, T. Nishio, and T. Oku, "Design and screening strategies for $\alpha$-Glucosidase inhibitors based on enzymological information," Current Topics in Medicinal Chemistry, vol. 9, no. 1, pp. 3-12, 2009.

[5] M. Balba, N. A. El-Hady, N. Taha, N. Rezki, and E. S. H. El Ashry, "Inhibition of $\alpha$-glucosidase and $\alpha$-amylase by diaryl derivatives of imidazole-thione and 1,2,4-triazole-thiol," European Journal of Medicinal Chemistry, vol. 46, no. 6, pp. 25962601, 2011.

[6] G.-L. Li, J.-Y. He, A. Zhang, Y. Wan, B. Wang, and W.-H. Chen, "Toward potent $\alpha$-glucosidase inhibitors based on xanthones: a closer look into the structure-activity correlations," European Journal of Medicinal Chemistry, vol. 46, no. 9, pp. 4050-4055, 2011.

[7] R. Pascale, A. Carocci, A. Catalano et al., "New N-(phenoxydecyl)phthalimide derivatives displaying potent inhibition 
activity towards $\alpha$-glucosidase," Bioorganic and Medicinal Chemistry, vol. 18, no. 16, pp. 5903-5914, 2010.

[8] B. C. Raju, A. K. Tiwari, J. A. Kumar et al., " $\alpha$-Glucosidase inhibitory antihyperglycemic activity of substituted chromenone derivatives," Bioorganic and Medicinal Chemistry, vol. 18, no. 1, pp. 358-365, 2010.

[9] H. W. Ryu, J. K. Cho, M. J. Curtis-Long et al., “ $\alpha$-Glucosidase inhibition and antihyperglycemic activity of prenylated xanthones from Garcinia mangostana," Phytochemistry, vol. 72, no. 17, pp. 2148-2154, 2011.

[10] S. J. Wang, J. F. Yan, X. Y. Wang, Z. Yang, F. W. Lin, and T. J. Zhang, "Synthesis and evaluation of the $\alpha$-glucosidase inhibitory activity of 3-[4-(phenylsulfonamido)benzoyl]-2H-1benzopyran-2-one derivatives," European Journal of Medicinal Chemistry, vol. 45, no. 3, pp. 1250-1255, 2010.

[11] W. D. Seo, J. H. Kim, J. E. Kang et al., "Sulfonamide chalcone as a new class of $\alpha$-glucosidase inhibitors," Bioorganic and Medicinal Chemistry Letters, vol. 15, no. 24, pp. 5514-5516, 2005.

[12] A. R. Katritzky, Z. Wang, M. Wang, C. R. Wilkerson, C. D. Hall, and N. G. Akhmedov, "Preparation of $\beta$-keto esters and $\beta$ diketones by $\mathrm{C}$-acylation/deacetylation of acetoacetic esters and acetonyl ketones with 1-acylbenzotriazoles," Journal of Organic Chemistry, vol. 69, no. 20, pp. 6617-6622, 2004.

[13] X. F. Zou, X. F. Jia, X. X. Wang, and G. Q. Xie, "Substitution of acyl for acetyl with $\mathrm{N}$-acylbenzotriazoles catalyzed by samarium triiodide," Synthetic Communications, vol. 37, no. 10, pp. 1617-1625, 2007.

[14] T. K. Morgan Jr., R. Lis, A. J. Marisca, T. M. Argentieri, M. E. Sullivan, and S. S. Wong, "Synthesis and cardiac electrophysiological activity of 2- and 3-[(substituted phenyl)alkyl] quinuclidines. Structure-activity relationships," Journal of Medicinal Chemistry, vol. 30, no. 12, pp. 2259-2269, 1987. 

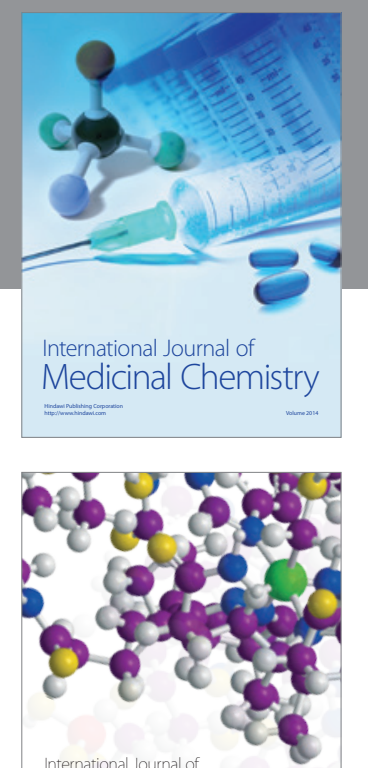

\section{Carbohydrate} Chemistry

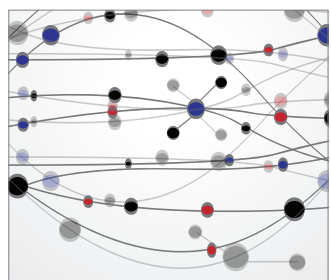

The Scientific World Journal
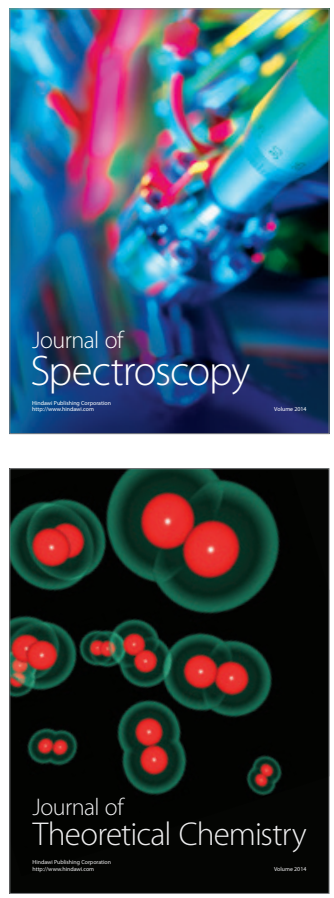
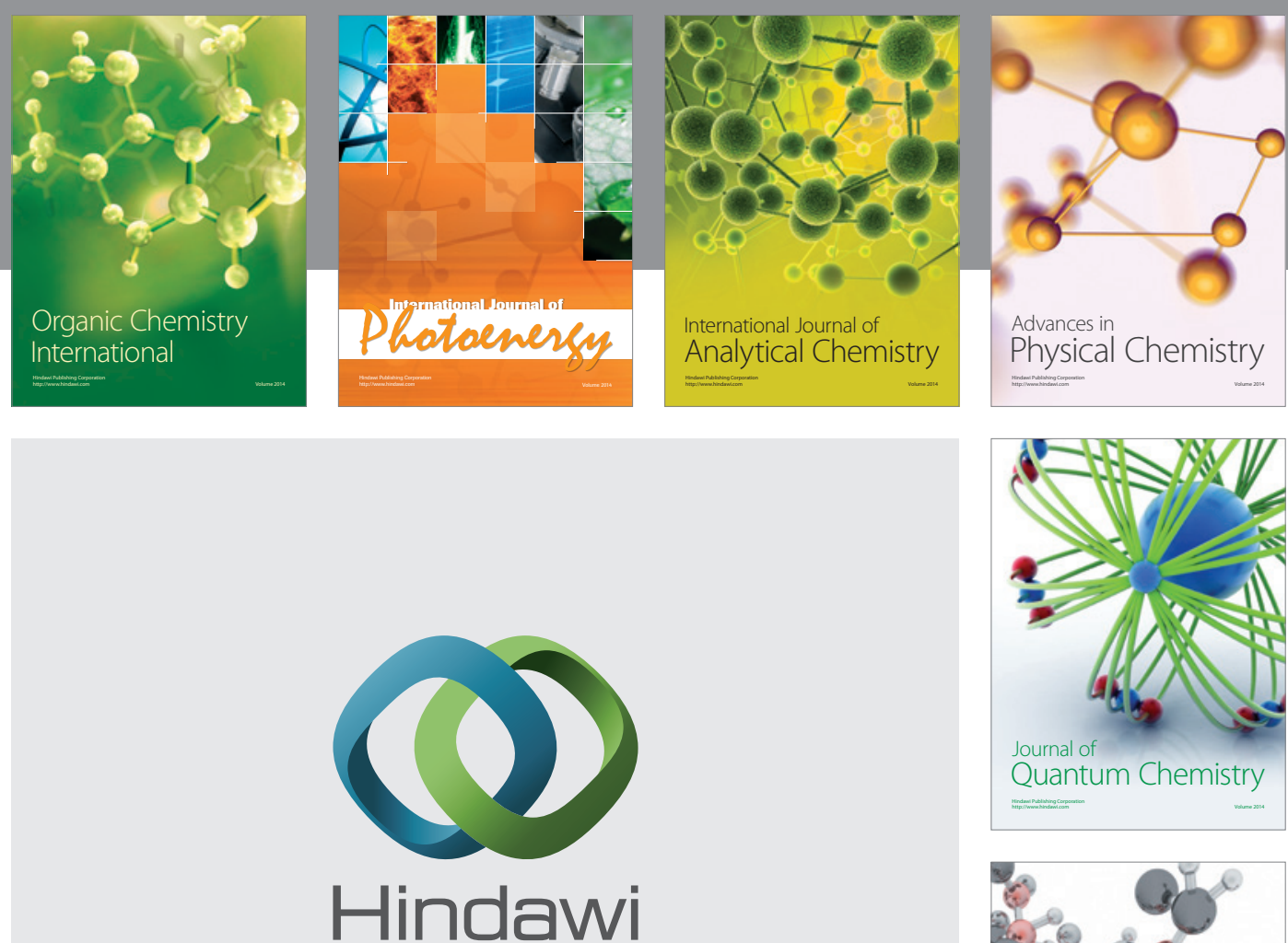

Submit your manuscripts at

http://www.hindawi.com

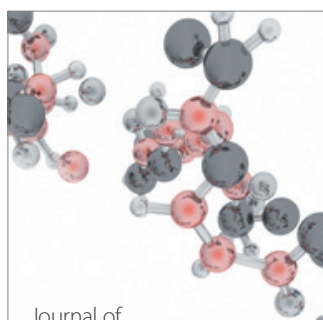

Analytical Methods

in Chemistry

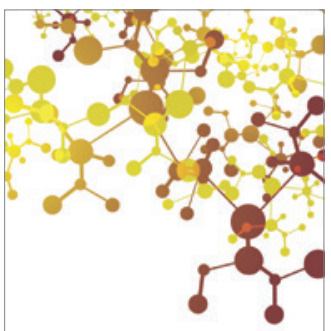

Journal of

Applied Chemistry

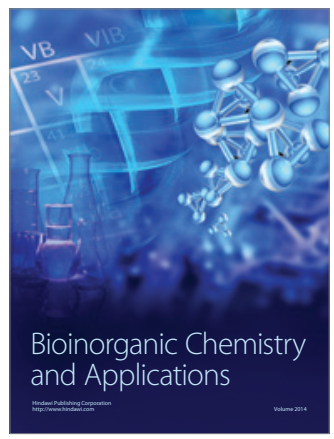

Inorganic Chemistry
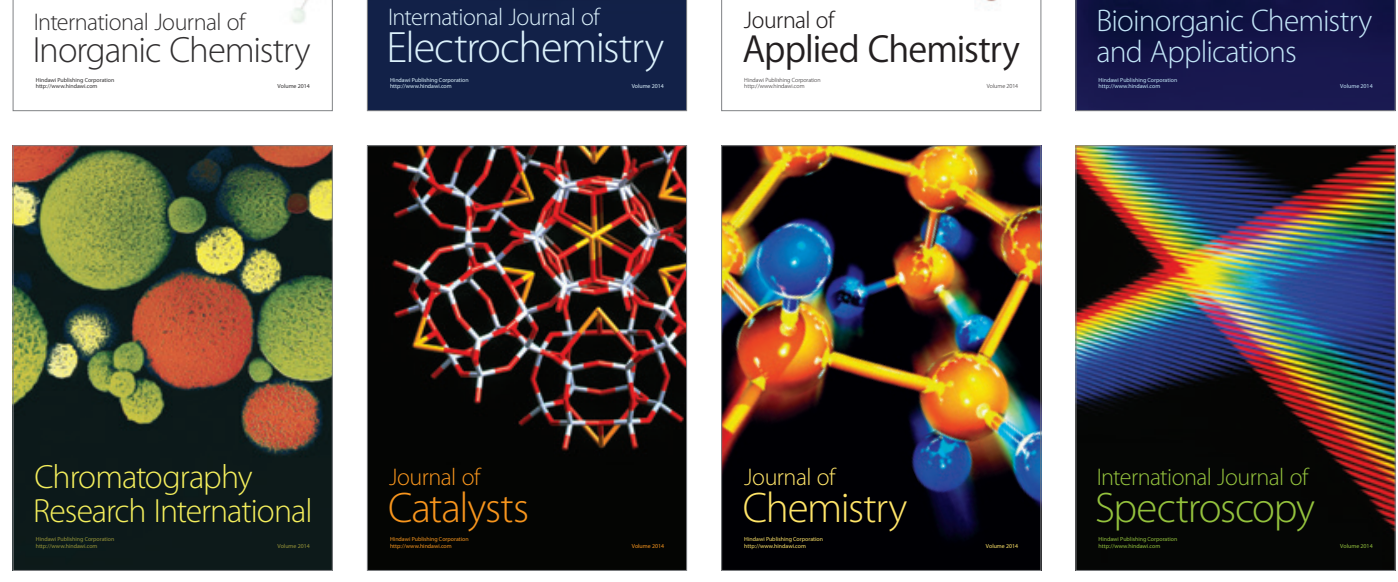